\title{
Chronic kidney disease: a risk factor for stroke and bleeding in patients with atrial fibrillation
}

A Danish study published in the New England Journal of Medicine reports that among patients with atrial fibrillation, those who also have chronic kidney disease (CKD) have an increased risk of systemic thromboembolism, bleeding and stroke.

Oral anticoagulant therapies such as warfarin are the most effective therapy to reduce the risk of stroke or systemic thromboembolism in patients with atrial fibrillation, but some researchers have suggested that warfarin might increase the risk of ischaemic stroke among atrial fibrillation patients who are also undergoing dialysis treatment.

"Patients with CKD are often excluded from clinical trials," says Jonas Bjerring Olesen, corresponding author on the Danish study. "The effect of antithrombotic treatment in the fragile group of patients with both CKD and atrial fibrillation has not been investigated in a large, randomized trial. Renal disease is considered a risk factor for bleeding in patients with atrial fibrillation and, although some previous studies have suggested that CKD increases the stroke risk, CKD has not been considered an established stroke risk factor in patients with atrial fibrillation."

The researchers used data from various Danish national registries to identify all patients who had been discharged from hospital with a diagnosis of nonvalvular atrial fibrillation during the 12-year study period between 1997 and 2008. They linked data from the Central Population Registry, the National Patient Registry, the Registry of Medicinal Product Statistics, the National Registry on Regular Dialysis and Transplantation, and the National Registry of Causes of Death, in order to determine diagnoses, surgical procedures undergone, drug therapies used, and outcomes of the individuals included in the study.

Olesen et al.'s study included data from 132,372 patients with nonvalvular atrial fibrillation. Among the included individuals, 127,884 (96.6\%) had no renal disease at baseline, 3,587 (2.7\%) had nonend-stage CKD and 901 patients (0.7\%) needed renal replacement therapy. Over the course of the study, 4,538 patients who did not initially have renal disease developed non-end-stage CKD, and 477 patients who did not initially have renal disease required renal replacement therapy at some point during the study. Data were not censored in relation to change in renal status, and were instead analysed according to the current renal status.

The researchers found that the risk of stroke or systemic thromboembolism was much higher in patients with nonend-stage CKD and in those on renal replacement therapy than among those without renal disease (hazard ratios 1.49 for non-end-stage CKD and 1.83 for those on renal replacement therapy).

Looking at the pharmacologic treatments that patients received, Olesen and co-workers found that warfarin treatment was associated with a significant decrease in the risk of stroke or systemic thromboembolism in the total population and in the subgroup of patients on renal replacement therapy, with a nonsignificant decrease in the risk among patients with non-end-stage CKD. The combination of warfarin plus aspirin was also associated with a decreased risk of stroke or systemic thromboembolism, although aspirin alone was associated with an increased risk of stroke or systemic thromboembolism in the total population and among those with any type of renal disease.

Olesen et al. also found that the risk of bleeding was higher in patients with non-end-stage CKD and in those requiring renal replacement therapy than it was in patients without renal disease. In addition, treatment with warfarin, aspirin, or both drugs was found to increase this bleeding risk. The risk of

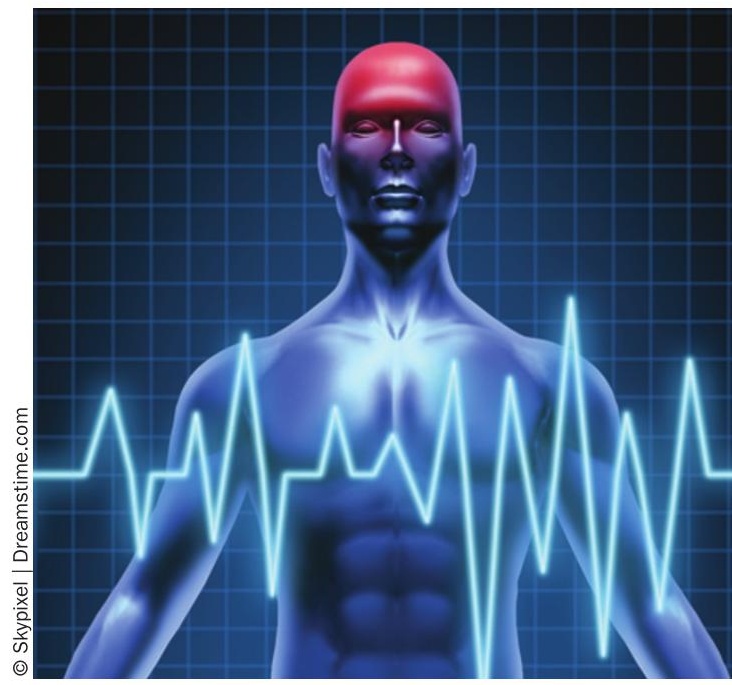

bleeding was found to increase with increased doses of loop diuretic among patients with non-end-stage CKD.

In addition, patients with renal disease (those not requiring and those requiring renal replacement therapy) were at increased risk of myocardial infarction and increased risk of any-cause death, compared with patients without renal disease (hazard ratios 2.37 for non-endstage CKD and 3.35 for CKD requiring renal replacement therapy).

"Establishing CKD as a risk factor for stroke (and not just a risk factor for bleeding) in patients with atrial fibrillation and confirming that warfarin is also effective in patients with CKD are novel findings," says Olesen. "The natural next step is to determine the net clinical benefit of warfarin in patients with both atrial fibrillation and CKD — that is, whether or not the beneficial effect of warfarin on the stroke risk is outweighed by the increased risk of bleeding."

Rebecca Kelsey

Original article Bjerring Olesen, J. et al. Stroke and bleeding in atrial fibrillation with chronic kidney disease. N. Engl. J. Med. 367, 625-635 (2012) 